\title{
Navigation of High Intensity Focused Ultrasound Applicator with an Integrated Three-Dimensional Ultrasound Imaging System
}

\author{
Ichiro Sakuma ${ }^{1}$, Yuichi Takai ${ }^{1}$, Etsuko Kobayashi ${ }^{1}$, Hiroshi Inada ${ }^{1}$, \\ Katsuhiko Fujimoto ${ }^{2}$, and Tekehide Asano ${ }^{3}$ \\ ${ }^{1}$ Graduate School of Frontier Sciences, ${ }^{2}$ Graduate School of Engineering, \\ The University of Tokyo. 7-3-1, Hongo, Bunkyok-ku, Tokyo 113-8656 Japan \\ sakuma@k.u-tokokyo.ac.jp, \\ \{takai,estuko, inadah\}@miki.pe.u-tokyo.ac.jp \\ ${ }^{2}$ Toshiba Medical Systems Company, 1385 Ogami, Ohtawara Tochigi 324-8550 Japan \\ katsuhiko.fujimoto@toshiba.co.jp \\ ${ }^{3}$ 2nd Department of Surgery, Chiba University School of Medicine, \\ 1-8-1, Inohana, Chuo-ku, Chiba City, Chiba 260-8670 Japan \\ asano@med.m.chiba-u.ac.jp
}

\begin{abstract}
A three-dimensional ultrasound imaging system was integrated in a HIFU applicator. This makes it easy to register the focal position of the HIFU applicator in the obtained three-dimensional volume data by three-dimensional ultrasound imaging system. The applicator was mounted on a mechanical manipulator with three degrees of freedom. The HIFU probe was positioned according to the volume data of the tissue around the target obtained by the ultrasound imaging system. A phantom study was conducted to evaluate the accuracy of navigation. Navigation errors were within $3 \mathrm{~mm}$. It could also detect the monitor the change in acoustic properties of tissue due to HIFU application. The system uses history of HIFU application during therapy can be recorded in the system to assist appropriate manipulation of the probe by surgeons. The developed system is a simple, a cost-effective, and compact device for minimally invasive liver surgery.
\end{abstract}

\section{Introduction}

Recently, several minimally invasive surgical techniques have been studied as an alternative to liver resection. These include chemical ablation by percutaneous injection of ethanol, radio-frequency microwave thermal ablation and thermal ablation by high intensity focused ultrasound (HIFU).[1-3] HIFU showed promising results in non invasive thermal ablation techniques of liver tumor. Since ultrasound can penetrate deep into soft tissue, there is no need for implanting any medical devices in a patient. MR imaging has been used for guidance and temperature monitoring of HIFU therapy [4-6]. MR imaging is powerful both for guide and monitor HIFU procedure. However, it requires expensive facilities. In this study, as an alternative to guide HIFU procedure, three-dimensional ultrasound imaging system was integrated in HIFU applicator. We integrated a three-dimensional ultrasound imaging system in a HIFU applicator. This makes it easy to register the focal position of the HIFU appli- 
cator in the obtained three-dimensional volume data by three-dimensional ultrasound imaging system. We also mounted it on a mechanical manipulator and positioned it based on obtained three-dimensional ultrasound images. We evaluated the accuracy of applicator positioning through phantom experiments.

\section{Integration of Three-Dimensional Ultrasound Imaging System to HIFU System}

\subsection{HIFU System [7]}

In this study, we used a high intensity focused ultrasound applicator consisting of 12 piezoelectric oscillators placed on the spherical base. Frequency if ultrasound wave was $1.65 \mathrm{MHz}$. The opening of the probe was $110 \mathrm{~mm}$ and the focal length of the HIFU applicator was $100 \mathrm{~mm}$. It is well known that formation of micro bubbles during ultrasound application due to cavitations interfere with ultrasound propagation in tissue. It leads to attenuation of focused ultrasound energy and dislocation of ablated area towards the HIFU applicator relative to its focal point. In this system, frequency modulation of applied ultrasound was used to suppress generation of cavitations. When the excessive power is concentrated at the very narrow area of the liver tissue, instantaneous destruction of tissue occurs leading to hemorrhage and formation of void in tissue. Thus moderate focusing of ultrasound power near the focal point is required. In this system, neighboring piezoelectric oscillators were driven in opposite phase. Interference between ultrasound waves caused moderate ablation in larger area near the focal point avoiding destruction of tissue structure.

\subsection{Three Dimensional Ultrasound Imaging System [8]}

A prove of the ultrasound imaging system (TOSHIBA PowerVision4000) was installed at the center of the HIFU applicator as shown in Figure 1. The distance between the ultrasound imaging prove and the focal point of the HIFU applicator was $78 \mathrm{~mm}$. The probe was equipped with a stepping motor with reduction gears. To acquire $3 \mathrm{D}$ images, we rotated the probe with the stepping motor. Space under the HIFU applicator should be filled with de-gassed water to enable ultrasound propagation. An o-ring was to seal the rotating part of ultrasound imaging system and HIFU applicator.

A sets of two-dimensional ultrasound image data were obtained at interval of the fixed angle when the probe was rotated at a fixed speed. The volume data could be reconstructed from sequence of 2D images. Because 2D images are obtained in sequence at interval of the fixed angle, the datasets are discrete. Thus we interpolated the vacant space between neighboring two images to obtain three-dimensional volume information. In this study, we measured 120 images in 180 degrees, consequently the resolution of this image was approximately $2 \mathrm{~mm}$ at the edge of a circle whose radius is $82 \mathrm{~mm}$. We used volume rendering as visualization method. However, since our system displays 3D image by volume rendering, the system does not have to execute boundary extraction and segmentation. In our system, the volume rendering graphic accelerator (VolumePro, Mitsubishi Electronics of America, MA. U.S.A.) enabled real time volume rendering in the conventional $\mathrm{PC}$ system. 


\subsection{IFU Probe Navigation System}

The HIFU applicator with integrated three-dimensional ultrasound imaging system was mounted on a mechanical arm having three degrees of freedom: $\mathrm{X}, \mathrm{Y}$, and $\mathrm{Z}$ direction. A stepping motor drove each axis. The total system configuration is shown in Figure 2. In this system, we integrated mechanically the ultrasound imaging prove and the HIFU applicator. Thus it is easy to register location of the focal point of the HIFU applicator in the acquired three-dimensional information of tissue around the target. We developed software to display the focal point of the HIFU applicator in the volume data or arbitrary cross sectional data obtained by the three-dimensional ultrasound imaging system as shown in figure 3. It can also record the history of applied ultrasound energy locations to assist intra-operative therapeutic planning of thermal ablation.

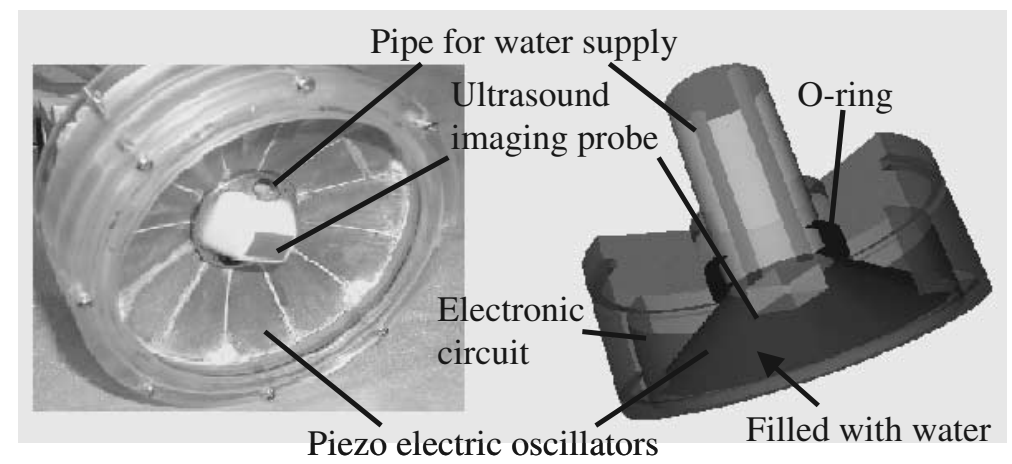

Fig. 1. HIFU applicator with an integrated three-dimensional ultrasound imaging system

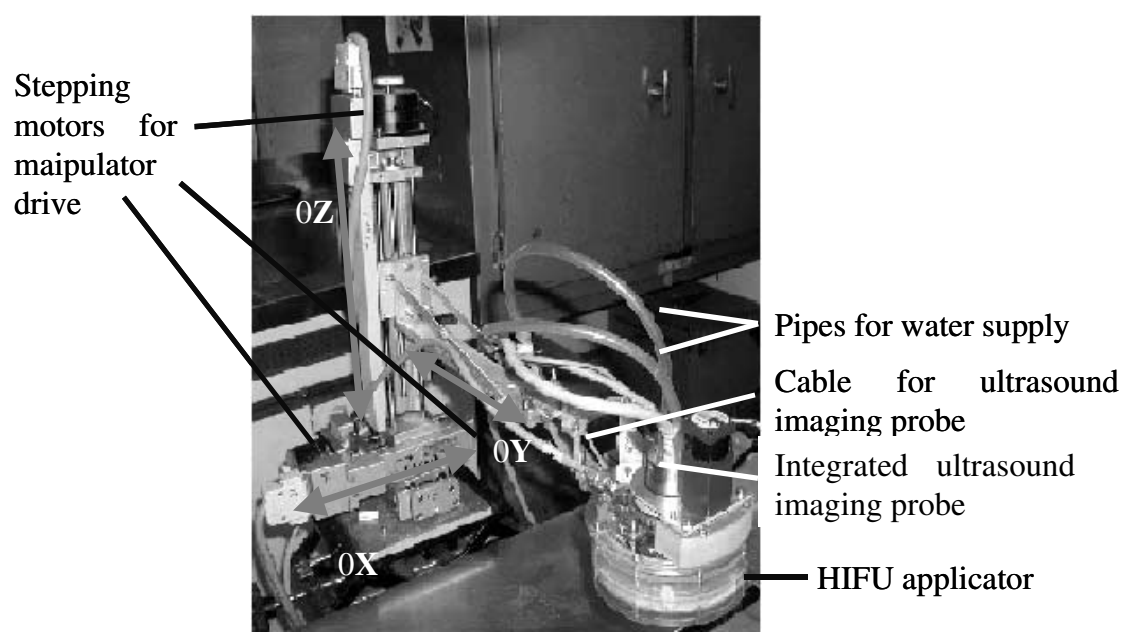

Fig. 2. HIFU applicator with an integrated three-dimensional ultrasound imaging system mounted on a manipulator with three degrees of freedom 


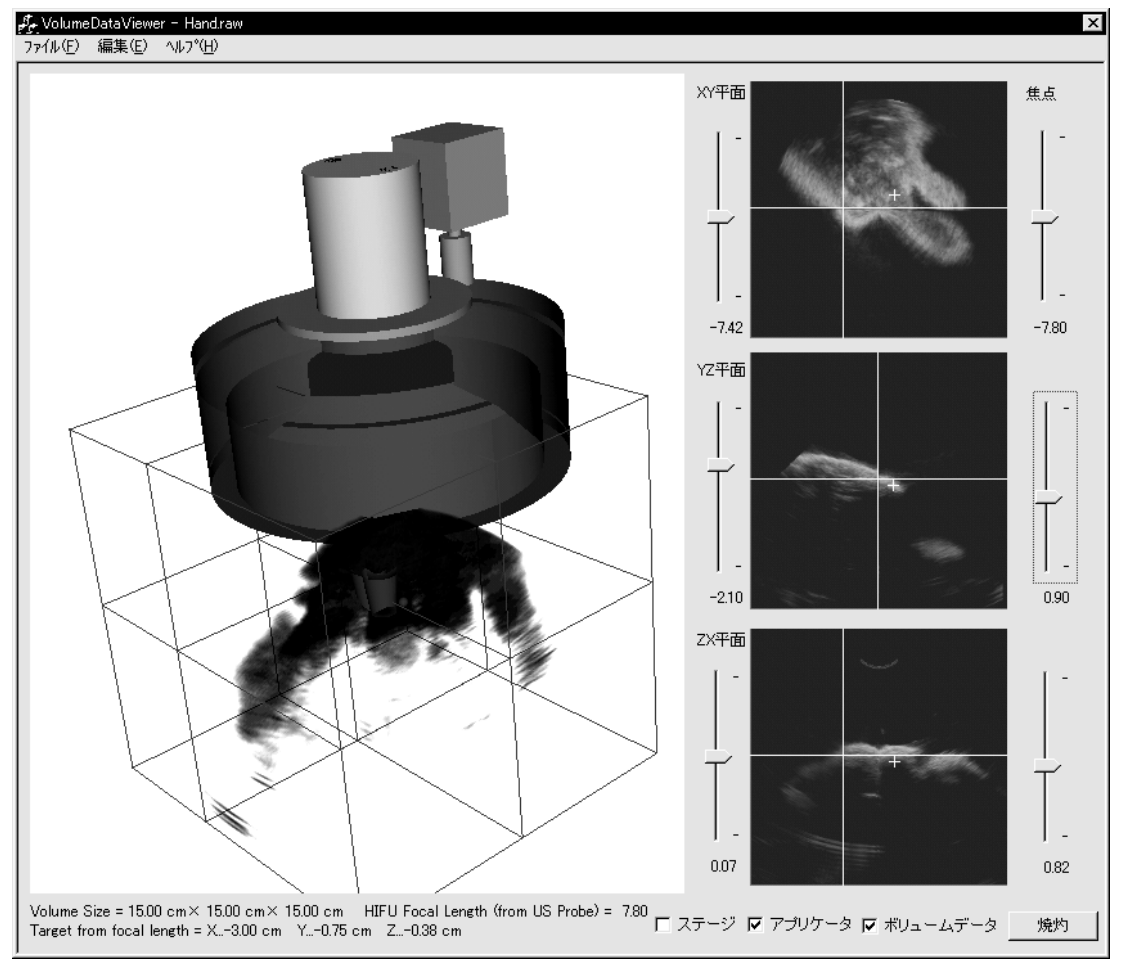

Fig. 3. An example of display for HIFU applicator navigation. In the right three panel arbitrary cross sections can be displayed together with focal point coordinate. In the left panel, reconstructed volume is displayed together with estimated heated region

\section{Results}

\subsection{Accuracy of the Three-Dimensional Ultrasound Imaging System}

To evaluate accuracy of the three-dimensional ultrasound imaging system, we have conducted the following experiment. A silicone phantom $(50 \mathrm{~mm} \times 60 \mathrm{~mm} \times 40 \mathrm{~mm})$ was placed in a water filled container with it upper surface perpendicular to the axis of ultrasound probe. The distance between the phantom and the probe was $90 \mathrm{~mm}$. Ultrasound image of the upper surface was obtained and length of each side was measured. Measurement error was within $3 \mathrm{~mm}$.

\subsection{Navigation of HIFU Probe}

\section{(1) Modeling of Heated Region by the HIFU Applicator}

Temperature sensitive phantom was made of thermal solidified polysaccharide, thermo-sensitive dye vinyl chloride, and water. The color of the phantom was red before heating. When the phantom was heated up to approximately $60{ }^{\circ} \mathrm{C}$, its color changed to white. We could determine the localization of the heated area in the phantom by identifying its color change. We placed the phantom (height: $40 \mathrm{~mm}$ ) under the HIFU applicator with distance of $80 \mathrm{~mm}$ and applied HIFU with power of 
120 and $150 \mathrm{~W}$ for 15 seconds considering usual condition in clinical evaluation of the same HIFU system. Using the obtained data, we simply modeled the area of heated with HIFU as conical shape region.

\section{(2) Phantom Experiment of Navigation of HIFU Applicator with Three-Dimensional Ultrasound Image}

Three metal bolts (diameter: $3 \mathrm{~mm}$, length: $40 \mathrm{~mm}$ ) were placed in a temperature sensitive phantom in a triangular configuration as markers for HIFU applicator positioning. Distance between bolts was set as long as $30 \mathrm{~mm}$. We tried to apply ultrasound energy in this triangular space surrounded by these three bolts. The locations of three bolts were identified by the three-dimensional ultrasound imaging system. The applicator was positioned so that HIFU applicators focal point was set at a certain distance from the tip of three markers using the mechanical manipulator. The heated area in the phantom was identified by change in color of temperature sensitive phantom. We applied $120 \mathrm{~W}$ of ultrasound energy for 15 seconds. We evaluated the distances between the center of gravity of the area where its color changed to white due to temperature rise and the planned focal position of the HIFU applicator. The result is shown in Figure 4. The distances between the planned focal point and actual center of gravity of the heated areas were $1.5,2.0,3.0 \mathrm{~mm}$ respectively.

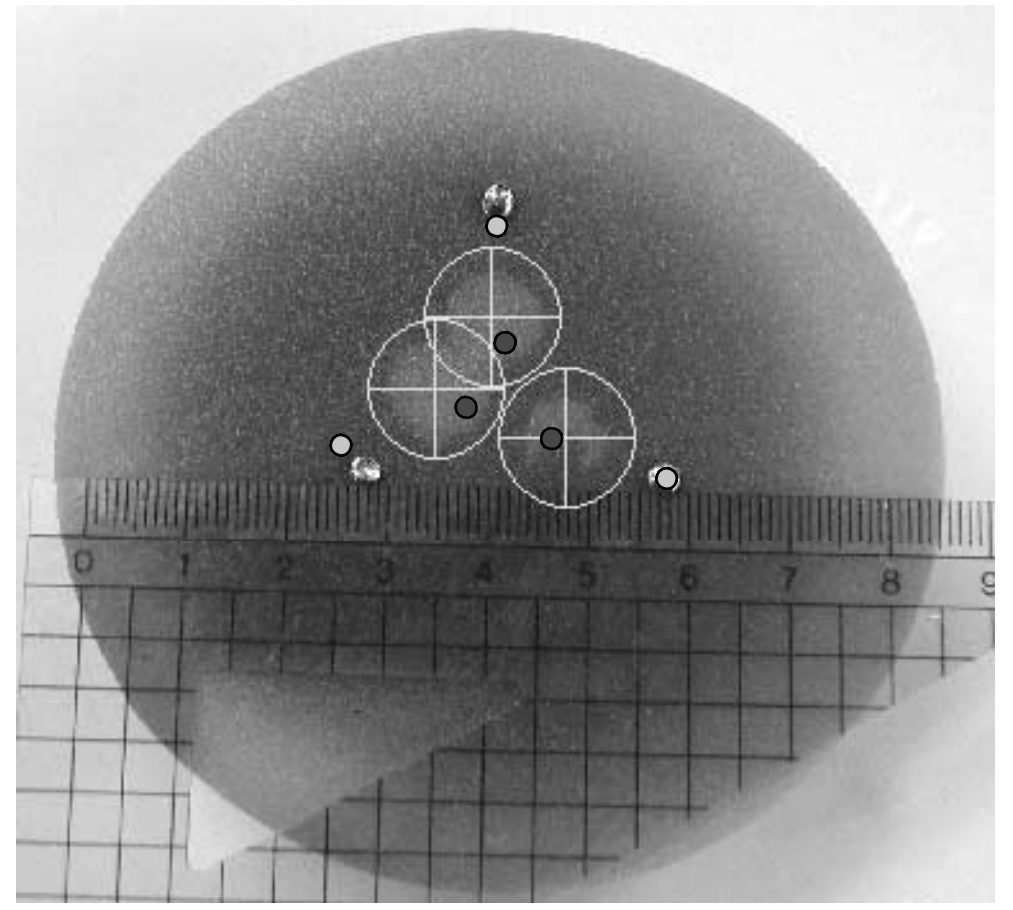

Fig. 4. Results of a phantom experiment

Dots near the marker bolts stand for measured position of markers by the three-dimensional ultrasound imaging system. Dots in a circle stand for planed focal position of HIFU and point on the cross in each circle stands for center of gravity of the heated region in the phantom. 


\subsection{Ultrasound Imaging of HIFU Ablation in Porcine Liver Sample}

We applied HIFU to a porcine liver sample. We obtained ultrasound threedimensional images before after application of HIFU. Figure 5 shows the image obtained after application of $120 \mathrm{~W}$ HIFU for 15 seconds. The optical image of the heated area is also displayed in the figure. We could identify the heated area as area with high contrast region. However, the contrast decreased as time elapsed after HIFU application.

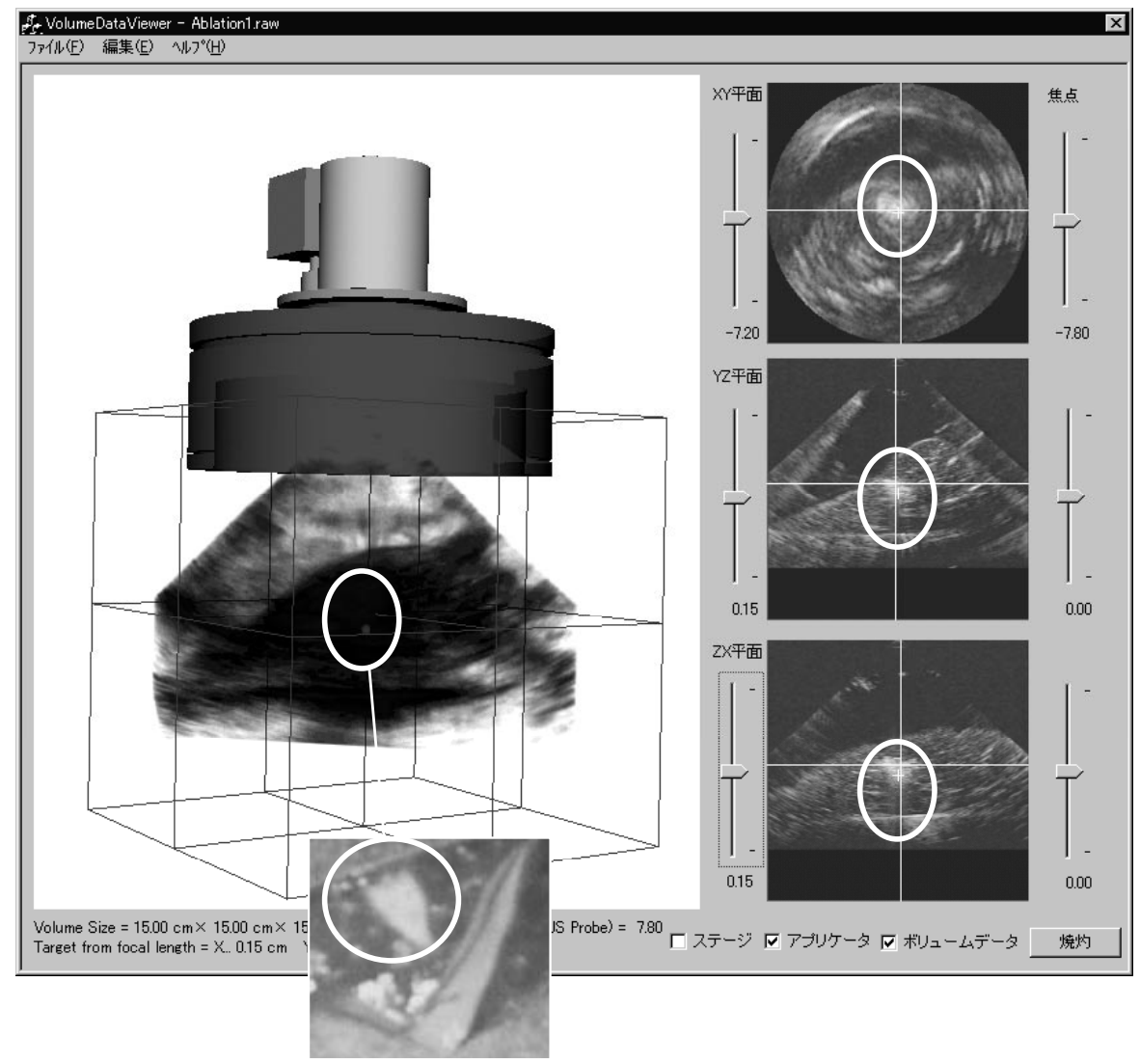

Fig. 5. Ultrasound images after application of $120 \mathrm{~W}$ HIFU for 15 seconds to porcine liver sample. High contrast region are circled in the figures. Actual optical image of the heated area is also shown

\section{Discussion}

We integrated three-dimensional ultrasound imaging system in a HIFU applicator. This made it easy to register the focal point of HIFU applicator in volume information of the target tissues. Davies et al proposed use of robot to position the HIFU applicator for neuro-surgery [9]. It still requires frame and position measurement system to 
localize the HIFU applicator. Since the developed system requires no additional devices for navigation, it is a cost-effective, simple, and compact device for minimally invasive liver surgery. Intra-operative monitoring of the change in acoustic properties of the tissue due to HIFU therapy and recording of the history of the HIFU application assist surgeon to manipulate appropriately HIFU applicator

Since the source of error inherent to the system is limited to mechanical alignment of HIFU applicator and ultrasound imaging system. The results in phantom experiment showed that the navigation error was within $3 \mathrm{~mm}$. This was mainly due to image resolution of the currently used ultrasound imaging system and errors in modeling the heated region by HIFU. Various computational models have been proposed for estimation of ablated area by HIFU therapy [10]. We will incorporate these theoretical models in navigation system to improve navigation accuracy.

\section{References}

1. Diederich CJ., Nau WH., Stauffer PR.: Ultrasound Applicators for Interstitial Thermal Coagulation, IEEE Trans. Ultrasonics, Ferrpelectrics and Frequency Control, 46 (1999) 12181227

2. Arefiev A., Chapelon JY, Tavakkoli J., Cathignol D.: Ultrasound-induced tissue ablation: studies on isolated perfused porcine liver, Ultrasound in Medicine and Biology, 24 (1998) 1033-1043

3. Vaezy S., Martin R., Caps M., Taylor S., Beach K., Carter S., Kaczkowski P., Keilman G., Helton S., Chandler W., Mourad P., Rice M., Roy R., Crum L.: Liver hemostasis using high-intensity focused Ultrasound, Ultrasound in Medicine and Biology, 23 (1997) 14131420

4. Daum DR., Smith NB., King R., Hynynen K.: In vivo Demonstration of Noninvasive Thermal Surgery of the Liver and Kidney Using a Ultrasnoic Phased Array, Ultrasound in Medicine and Biology, 25 (1999) 1087-1098

5. Chen L., Bouley D., Yuh E., C'Arceul H., Butts K.: Study of Focused Ultrasound Tissue Damage Using MRI and Histology, J. Magn. Reson. Imaging 10 (1999) 146-153

6. Chung A., Hynynen K., Cline HE., Colucci V., Oshio KM, Jolesz F.: Optimaization of spoiled gradient-echo phase imaging for in vivo localization of focused ultrasound beam. Magn Reson Med, 36 (1996) 745-742

7. Asano T., Ito T., Sugamoto Y., Kondo S., Takayama W., Kenmoch T., Maruyama M., Miyauchi H., Mitsui Y., Ochiai T., Kainuma O., Tokoro Y., Jingu K., Nakagori T., Yamamoto H., Fujimoto K., Satou K.: Cancer Therapy by focused ultrasound, Geka 62 (2000) 1689-1695

8. Sakuma I., Tanaka Y., Takai Y., Kobayashi E., Dohi T., Schorr O. Hata N. Iseki H., Muiragaki Y., Hori T., Takakura K.: Three dimensional digital ultrasound imaging system for surgical navigation, Proc. CARS2001 (2001) 118-123

9. ,Davies BL., Chauhan S., Lowe MJS.: A Robotic Approach to HIFU Based Neurosurgery, LNCS 1496 (1998) 386-396

10. Bortros YY., Volakis JL., VanBaren P., Ebbini ES.: A hybrid computational model for ultrasound phased-array heating in presence of strongly scattering obstacles. IEEE Trans Biomed Eng 44 (1997) 1039-1050 